\title{
New financial spaces drawn by institutional regulatory barriers. What effects on territorial sustainable development?
}

\author{
Isabella Varraso, Oriana Cesari ${ }^{(1)(2)}$
}

\begin{abstract}
Financial flows affect sustainability. Financial regulation has a central role in sustainable development of regions. Recent crisis of 2008 has shown how financial space equilibrium is entangled with economic stability of territories so that changes in financial space can transform local and global financial conditions.

Considering the need to develop sustainable relations between financial space and real economy, the aim of this article is to examine, from a geographical perspective, how international institutions may create or reduce, through regulatory mechanism, barriers to the effects of uncontrolled financial flows.

Reproducing spaces involved and drawing results using cartography, poles and barriers are considered. As result, a space is outlined where is possible to study future diffusion patterns and consider if the barrier system observed reveals to be absorbing in the sense that financial contagion prevention is achieved with a significant reduction of risk, or if it is not absorbing so that risk shifts to lower levels of geographical scale reaching for the intersections between financial space and everyday economic life of territories involved.
\end{abstract}

Key Words: Financial space, Financial flows, Financial Geography, International Institutions, Regulatory barriers

\section{Premessa}

Già nel 2001 il geografo Thrift osserva che "the 'financialization' of the economy ... had become so noticeable that it was no longer possible to envisage money and finance as a subsidiary activity whose role was simply to oil the wheels of production" (p. 5655) e più recentemente Dixon (2011) nota che "finance seems to pervade almost all aspects of contemporary economic activity, from the way firms are managed to the built environment of urban centers" (p. 851).

I geografi sempre attenti, per epistemologia disciplinare, ai cambiamenti spaziali causati dai fenomeni anche economici, dagli anni Ottanta, stanno studiando con sistematicità questi processi, che stanno modificando le dinamiche dei sistemi territoriali, e hanno prodotto "a series of works on money and finance which has proved the value of a distinctive geographic perspective that emphasizes how space creates money, as well as how money creates space" (Thrift, 2001, p. 5655). Hanno dimostrato, fra l'altro, che i sistemi finanziari non sono neutrali rispetto alle strutture spaziali sulle quali agiscono, ma influenzano le ubicazioni dei servizi e degli istituti di credito e i flussi di capitali, di finanziamenti e di crediti fra aree.

Isabella VARRASO è ordinario di Geografia politico-economica, Dipartimento di Economia, Università di Foggia. Oriana CESARI è Dottore di Ricerca in "Economia e Tecnologie per lo Sviluppo Sostenibile", Dipartimento di Economia, Università di Foggia.

2 Il contributo è frutto del lavoro congiunto degli Autori. Ma a Isabella Varraso sono da attribuire i paragrafi 1 e 2 , a Oriana Cesari i successivi. 
Peraltro, i rapidi cambiamenti dei processi finanziari, verificatisi soprattutto nel mondo occidentale in seguito alla globalizzazione e allo sviluppo delle nuove tecnologie informatiche e delle comunicazioni, hanno fatto ritenere che il peso dei luoghi nelle scelte economico-finanziarie si fosse ridotto, tanto da far parlare alcuni studiosi di "fine della Geografia". Tuttavia, molte ricerche in questi anni hanno dimostrato che luoghi e regioni ancora influenzano e sono influenzati da ubicazioni di servizi, transazioni, interventi di istituti di credito localizzati, vincoli istituzionali e scelte statali (Martin, 1994; Agnes, 2000).

La Geografia guarda i flussi finanziari, anche alla luce dei processi di globalizzazione, considerando le funzioni che svolgono nelle diverse aree che attraversano e le forme che assumono nei sistemi finanziari in cui si muovono, analizzandoli alle scale sovra-nazionale, nazionale e regionale. Considera le modalità di impatto dei flussi sui territori, che sono diversi per configurazione fisica, densità e caratteri antropici, posizione geo-economica, livello di sviluppo e capacità organizzativa dei gruppi umani che li abitano.

Il sistema finanziario, come componente centrale di ogni economia di mercato, agevola "the allocation and deployment of economic resources, temporally and spatially, under conditions of risk and uncertainty. Consequently, understanding the form and functions of finance and financial systems across space and time is key to understanding the uneven geography of capitalism from the local to the global' (Dixon, 2011, p. 851).

Alle diverse scale, quindi, il passaggio dei flussi e le scelte finanziarie dei gruppi umani si ripercuotono sui territori favorendo o inibendo processi di crescita e di sviluppo. L'ingegnosità dei gruppi umani, nell'intersecare i flussi finanziari e gestirli, li mette in grado di intervenire opportunamente nelle proprie scelte spaziali e di adottare comportamenti economici che, ai diversi livelli spaziali d'intervento, producono processi di sviluppo sostenibile, e quindi rispettosi dell'ambiente e delle risorse per le generazioni future.

Il territorio, pertanto, è il punto di partenza e di arrivo dello sviluppo economico in quanto comprende tutte le forme di organizzazione dei gruppi umani, con i suoi inevitabili riflessi in termini di pianificazione, più o meno consapevole e voluta. Si potrebbe quasi dire che si ha sviluppo economico se a questo corrisponde sviluppo territoriale. E' una visione geo-economica che affronta le dinamiche di crescita e di sviluppo guardando: ai flussi - e quindi agli spostamenti nello spazio di persone, beni, capitali - in base ai quali stabilire le strategie idonee per promuovere i differenti livelli e indagare le eventuali ricadute in rapporto a ciascun contesto di riferimento; alle connotazioni spazio-temporali in cui si osservano e si manifestano; alle realtà ambientali, e quindi anche socio-culturali, in cui il gruppo umano vive e opera (Varraso, 2004). Inoltre, il crescente affermarsi di cultura ed etica ambientali, sensibili ai nuovi rischi insiti nelle varie forme di squilibri, soprattutto naturali, intensifica ulteriormente la riflessione geografica, già tradizionalmente attenta a questi aspetti, verso i principi della sostenibilità.

Rimangono, ovviamente, ancora aperti i problemi di interpretazione economica dello sviluppo connessi alle difficoltà di considerare in modo convincente il carattere relativo del concetto e di parametrarne i diversi livelli, anche in relazione agli aspetti di sostenibilità.

Peraltro gli studiosi di processi finanziari riconoscono che si sono poco occupati 
di sviluppo sostenibile e cominciano a sollecitare l'opportunità di includere nelle loro indagini anche aspetti economici, sociali e ambientali in grado di declinare criteri di sostenibilità (Sudana, 2015).

Le istituzioni finanziarie svolgono in questa direzione un ruolo fondamentale per indirizzare le politiche e pianificazioni territoriali e fornire gli indirizzi di integrazione alle diverse scale operative. L'attuale crisi economico-finanziaria ha attribuito proprio alle istituzioni finanziarie, insieme a quelle politiche, la responsabilità di tracciare soluzioni condivise. Le iniziative in tal senso hanno evidenziato le differenze fra gli Stati, facendo emergere la complessità delle posizioni fra i governi sovrani e i limiti di integrazione fra le diverse regioni economiche.

In particolare nell'Unione Europea la crisi ha sollecitato la necessità di stabilire norme e indicazioni di governance finanziarie comuni, ma contemporaneamente ha fatto riemergere problematiche di confini fra stati e fra comunità a causa delle particolari convinzioni e condizioni socio-economiche dei singoli gruppi umani che li compongono e che, come noto, sono diversi non solo all'interno dell'Eurozona, ma anche fra le sue regioni e subregioni. Malgrado ciò, l'esigenza fortemente sentita di stabilire nuovi vincoli e confini fra le comunità ancora 'distanti' e il bisogno di una reale integrazione europea che consenta di affrontare e superare le attuali difficoltà economiche e politiche stanno effettivamente cambiando la geografia finanziaria dell'Unione Europea. Si sta meglio comprendendo che non sono solo i confini amministrativi a rappresentare vincoli e resistenze alle prospettate soluzioni ma le 'distanze culturali' (Sander, Kleimeir, Heuchemer, 2016) fra i Paesi europei. E sono proprio queste distanze, insieme a tutte le altre 'distanze percepite', che costituiscono barriere, o al contrario ponti, fra i diversi gruppi umani (Fiori, 1989 e 2012) e sulle quali occorre lavorare per costruire una integrazione condivisa perché percepita come utile, rispettosa delle identità ed efficace nell'azione. A tal fine criteri di etica e di sostenibilità sono decisamente auspicabili per ridurre le divergenze e le disuguaglianze.

In questa sede, considerando lo spazio finanziario configurato in nodi e poli, si considera il nuovo assetto istituzionale, con particolare riferimento al sistema di vigilanza bancaria internazionale ed europeo, tenendo presente come non sia ancora chiaro ad oggi quali effetti possa avere la regolamentazione finanziaria sullo sviluppo sostenibile (Graeme, 2016). In particolare, si esaminano in prospettiva geografica se vi possono essere, e quali manifestazioni spaziali possono assumere, gli effetti diretti della regolamentazione finanziaria sullo sviluppo sostenibile dei territori e se le previsioni di vigilanza, oltre a rendere più stabile lo spazio finanziario e i suoi nodi, possono avere una funzione di barriera alla diffusione dei contagi finanziari laddove tali nodi insistono.

\section{Flussi e barriere dello spazio finanziario europeo}

Transazioni virtuali e altri flussi finanziari in generale con i rispettivi network definiscono spazi di circolazione che, in quanto portatori di interessi legati alla finanza, definiscono spazi finanziari che si comportano diversamente in base alle loro funzioni e che si articolano fra loro intersecandosi con gli spazi dell'economia reale e con le concrete organizzazioni delle regioni.

Sono flussi che pur oltrepassando i luoghi si incontrano in nodi reali, i luoghi di 
azione, agganciando così i territori e facendosi da loro intercettare. Per cercare di raffigurarli nella loro complessità e nelle numerose interrelazioni che 'disegnano' e 'ridisegnano' l'economia globale si può pensarli organizzati in strutture di network, spesso gerarchiche, che agiscono a scale spaziali diverse e che si collegano a luoghi diversi per funzioni e per capacità di controllo del territorio, seguendo logiche transcalari oltre che multiscalari in base alla loro pervasività e significatività. Direttamente e/o indirettamente, agiscono sull'economia reale, incidendo nella vita dei gruppi umani e influenzando le organizzazioni regionali.

In questa prospettiva, la configurazione dello spazio finanziario può essere rappresentata da una struttura composta da nodi, flussi e barriere (fig. 1). Tale spazio finanziario, in analogia con l'ambiente fisico e le aree culturali, si estende sia all'interno che all'esterno dei propri confini. È caratterizzato dalla variabilità della sua struttura in funzione, da un lato, del suo grado di permeabilità ai flussi e, dall'altro, delle caratteristiche e dell'intensità dei processi che lo attraversano, oltre che dalla capacità attrattiva dei nodi e dalla forza dei collegamenti che li legano tra loro e con altri a loro funzionalmente contigui.

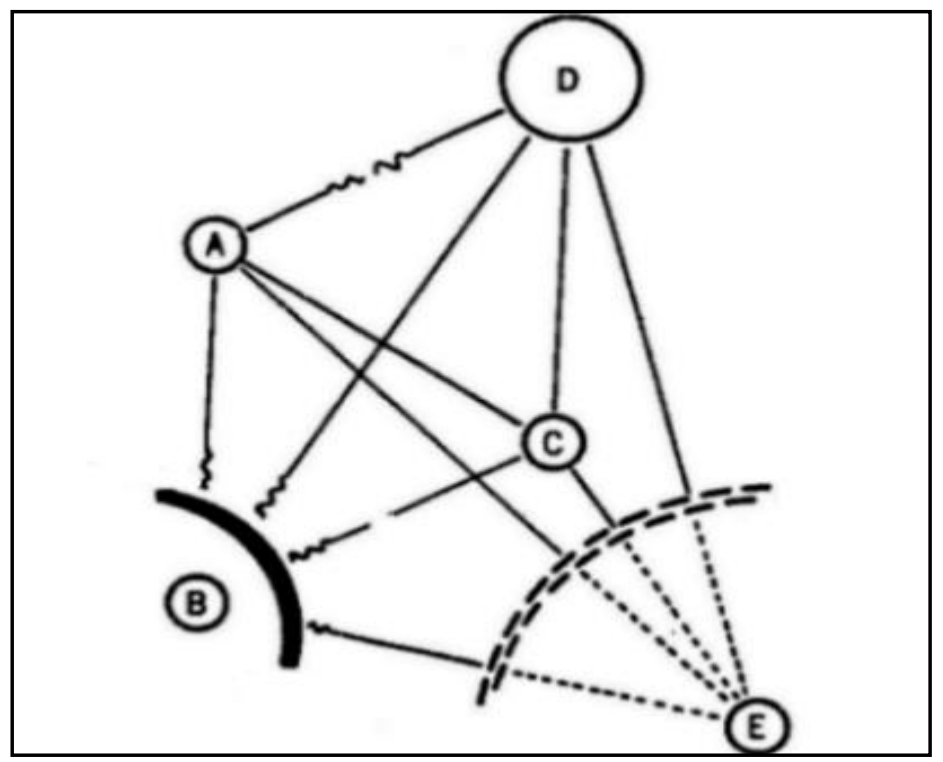

Fig. 1 - Struttura geografica di network composta da nodi ( punti nel grafico), flussi ( $i$ collegamenti fra $i$ nodi) $e$ barriere (le linee dal doppio tratteggio) (fonte: Gould, 1969, con lievi modifiche) che si possono cartograficamente 'sourapporre' alla base fisica del territorio deformandola (Bissanti, 1974).

Un'espressione concreta di questo modello e di questa logica è illustrata da Paltalidis et Al. (2015) che dimostra, attraverso la struttura di network elaborata dagli studiosi e riportata in fig. 2 , come può propagarsi il contagio nelle reti finanziarie europee considerando in particolare il network di Interbank Loan Market. 


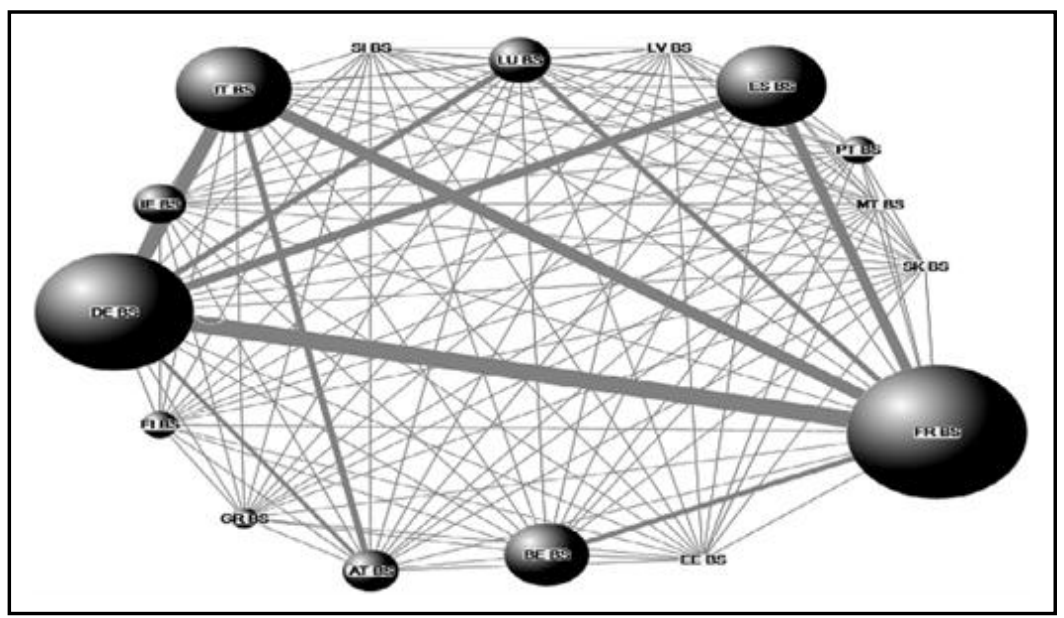

Fig. 2 - "The graph presents the network structure for the Interbank Loan Market cross-banking exposures. The nodes are the Banking Sectors for each euro area country. The links are the actual exposures from the Bank of International Settlements statistics and the different strength of the arrows and the links represents the different volumes (sizes) and the difference in the degree of interconnections for the bilateral interlinkages" (in Paltalidis et Al., 2015, p. 540).

Inoltre, i collegamenti fra nodi si possono interpretare come assi e 'corridoi' di propagazione di informazioni e di operazioni positive e virtuose o, al contrario, di diffusione di rischi e di contagi. Come per le vie di comunicazione fisiche, "cities are the nodal points in the organization, management and structuring of these networks. The main nodal points - the large cities and the urban networks ... - are interconnected via a dense physical and virtual network of international infrastructures. The nodal points linked by these corridors face the dialectical opposition between the space of flows and the space of places (Castells, 1996). The space of flows is based on a network that links up specific places with well defined social, cultural, physical and functional characteristics" (Albrechts, Coppens, 2003, p. 215).

Lo spazio che si osserva in tal modo non è uno spazio geometrico astratto, come in fig. 2, ma agisce su una realtà specifica e interviene a modificarla. Pertanto, la sua manifestazione non corrisponde più alla tradizionale base fisica ma l'area viene come 'ricostruita' dalle nuove relazioni che si creano e che intervengono a modificare il significato di confini e i ruoli di centralità delle aree coinvolte (Varraso, 2009). Come appare bene nella fig. 3 che mostra le nuove relazioni territoriali conseguenti ad un caso di studio "focusing on the high speed train (HST) terminal in Brussels as elabo-rated in the INTEREG IIC project CORRIDESIGN. This project selected seven megacorridors, of which three cross Brussels: the links with (1) Randstad, (2) RheinRuhr and (3) Lille/Paris/London" (Albrechts, Coppens, 2003, p. 215).

L'esempio fa anche comprendere come i confini tradizionalmente intesi debbano essere reinterpretati nel momento in cui nuove infrastrutture, sia fisiche sia virtuali, intervengono a modificare l'andamento dei flussi. Nel caso in oggetto si tratta di flussi materiali, ma la stessa logica e le stesse manifestazioni spaziali seguono i flussi immateriali come quelli finanziari. 


\section{A VISION FOR NORTH WEST EUROPE}

An agenda for a sustainable and balanced development

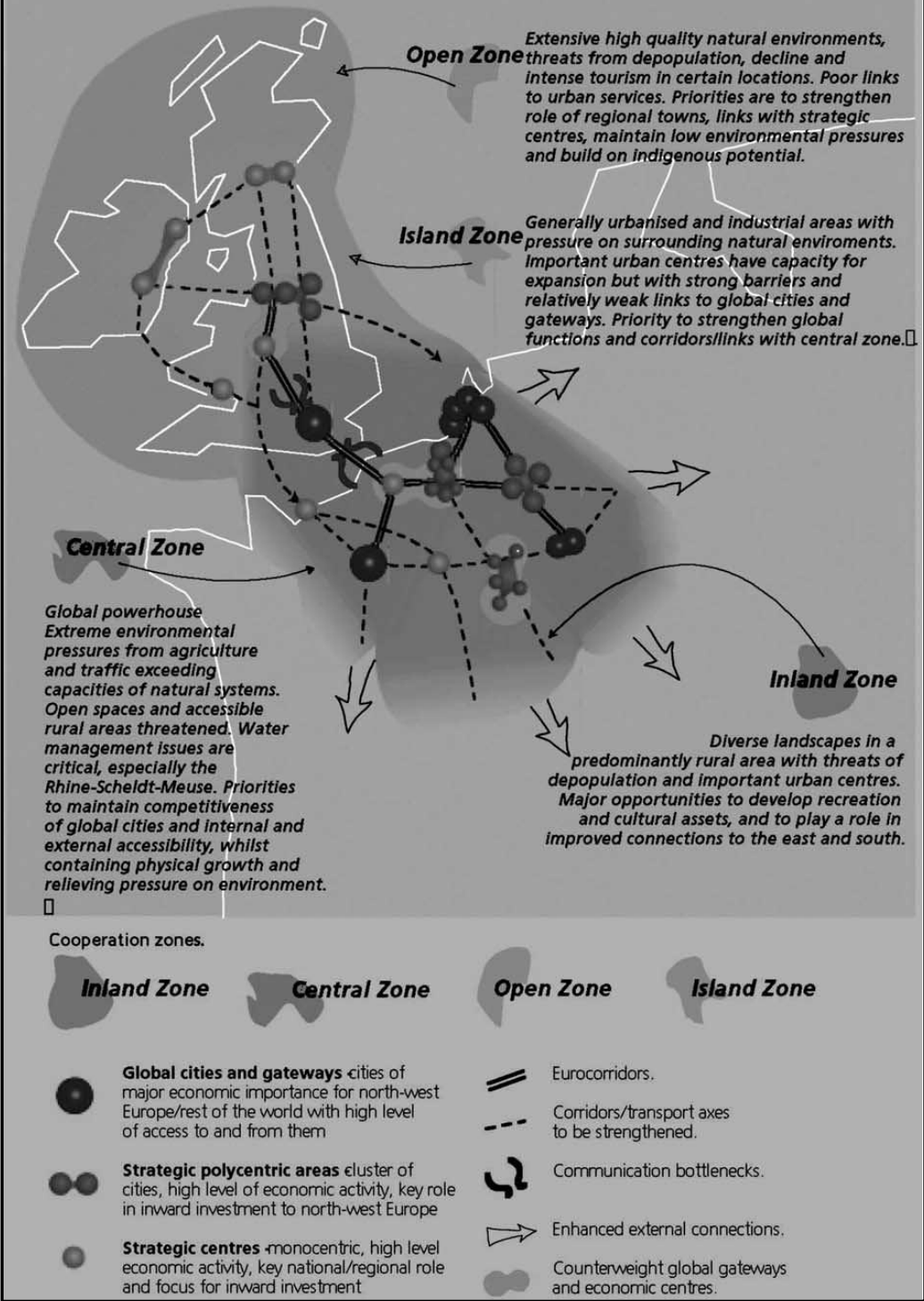

Fig. 3 - Rappresentazione del 'Vision Diagram' per l'Europa di Nord-Ovest in base al progetto “CORRIDESIGN" (Fonte: NWE Spatial Vision Group, 2000, in Albrechts, Coppens, 2003, p. 217).

Dalla figura 3 emergono, fra l'altro, le interazioni fra gli interventi sul territorio e gli attori sociali che "disegnano" uno spazio di relazioni dinamiche in grado di intervenire e trasformare le barriere sia naturali sia amministrative, costruendo nuove regioni di azione. Quindi, i vincoli che agiscono come confini sono connessi anche alle scelte degli 
attori sociali, politici ed economici e alle prescrizioni degli Stati nazione che ancora svolgono un ruolo importante nell'Unione Europea. Il peso diverso dei confini amministrativi e delle posizioni geografico-politiche, tuttora presente in Europa, è evidente anche solo considerando la rappresentazione cartografica in fig. 4 che mostra l'attuale estensione dello spazio monetario e finanziario europeo e che, come noto, è il risultato di diversi processi, fra cui la cooperazione fra gli Stati.

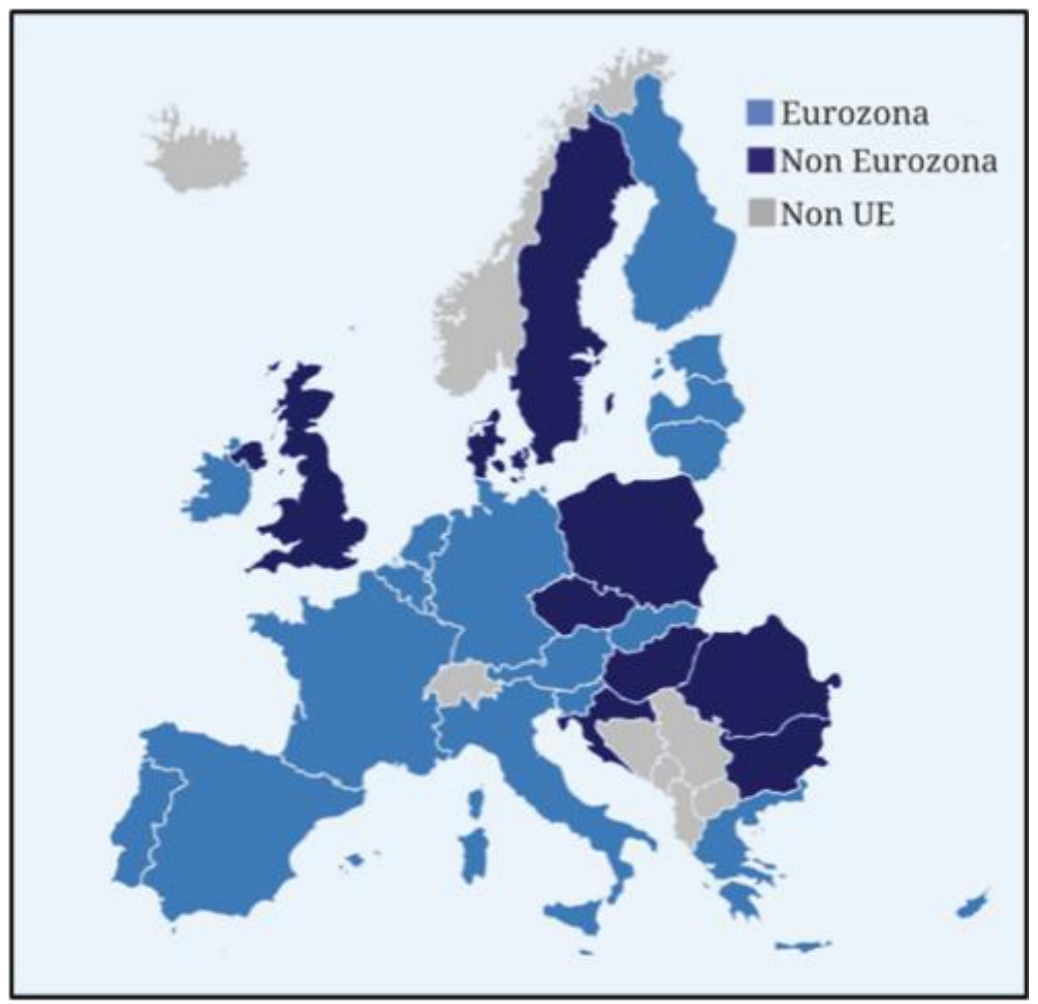

Fig. 4 - Carta amministrativa dell' Eurozona

(fonte: BCE, 2015, www.ech.europa.eu/ euro/intro/ html/map.it.html, con lievi modifiche).

In particolare, dall'insieme dei Paesi che adottano l'euro come valuta ufficiale, entro i confini dell'Unione Europea, si è formata l'Eurozona cui fanno parte: l'Austria, il Belgio, Cipro, l'Estonia, la Finlandia, la Francia, la Germania, la Grecia, l'Irlanda, l'Italia, la Lettonia, la Lituania, il Lussemburgo, Malta, i Paesi Bassi, il Portogallo, la Slovacchia, la Slovenia e la Spagna. Altri Stati dell'Unione europea che invece non hanno adottato l'euro sono: Bulgaria, Croazia, Danimarca, Polonia, Repubblica Ceca, Romania, Svezia, Ungheria e Regno Unito. Quest'ultimo potrebbe cessare di far parte anche dell'Unione europea a seguito del procedimento di conferma del recente risultato del referendum sulla Br-exit.

Le aree in fig 4, pur nella loro staticità, già danno una indicazione dei problemi di convergenza, di integrazione, e di adozione di politiche monetarie e finanziarie comuni, e 
rendono evidenti la diversa permeabilità dei confini che spesso comportano differenti scelte di politica economica. Anche all'interno dei confini dell'Eurozona, che pure definisce una precisa regione economica e monetaria, si potrebbero analogamente visualizzare differenti posizioni amministrative e vincoli normativi. Tuttavia i flussi si muovono nell'attuale "quadro di globalizzazione economica planetaria in cui i flussi finanziari internazionali, quelli energetici e di merci svuotano e rendono spesso inefficaci i poteri degli Stati nazionali di determinare la vita economica interna" (Caselli, 2014). Quindi, come si è già visto in fig 3 , le logiche che favoriscono o limitano i flussi trasformano le condizioni di confini fra gli Stati rendendoli più o meno permeabili alle dinamiche finanziarie e modificando gli spazi finanziari.

\section{Contagio finanziario, barriere istituzionali nell'Unione bancaria europea e nuovo sistema di vigilanza}

La configurazione dello spazio finanziario è il risultato della combinazione di molteplici processi di scambio che hanno origine a diversi livelli della scala geografica. Ne risultano, come detto, flussi che, nel loro percorso, attraversano il tracciato dei confini degli Stati, e delle Unioni, raggiungendo sempre più spesso altre longitudini e altri continenti. Nello spazio finanziario così definito si possono individuare, in particolare, nodi in cui hanno luogo attività finalizzate allo scambio delle cosiddette informationally sensitive securities, ovvero strumenti finanziari che necessitano per essere sviluppati della concentrazione di intermediari capaci di far convergere e aggregare informazioni specializzate e altamente qualificate (Kindleberger, 1974). Tali strumenti finanziari, standardizzati, possono diffondersi nello spazio a partire dai nodi, da cui possono trasferirsi, con distinti gradi di intensità, oltre i confini dell'area in cui hanno avuto origine, in funzione della presenza o meno di fattori ambientali e istituzionali in grado di intercettarli o alterarne il percorso. Tali fattori possono essere rappresentati come barriere capaci di arginare o, al contrario, di essere attraversate dai flussi, o ancora di deviarne la direzione (Gould, 1969, fig. 1).

La crisi finanziaria, iniziata nel 2007, può essere un esempio dell'applicazione di questo modello ad un concreto processo di diffusione. Lo shock generato dal fallimento e dalle perdite patrimoniali di alcune banche, infatti, si è propagato attraverso lo spazio finanziario, alterando gli equilibri dell'economia reale di diverse regioni ed evidenziando la capacità dei flussi finanziari di attraversare i confini degli Stati e di superarne le barriere di regolamentazione. Ciò ha comportato, fra l'altro, l'esigenza di opportune iniziative mirate.

Le istituzioni finanziarie internazionali si sono mosse in tal senso anche condizionando i processi in atto negli Stati e nei mercati (Gordon, 2015), tuttavia la normativa di vigilanza si è rivelata inefficace nell'intervenire adeguatamente sui rischi di contagio e si è reso necessario un rafforzamento della struttura dei controlli su banche e mercati finanziari.

Gli Stati dell'Unione Europea hanno attivato un sistema di barriere regolamentari per contrastare il contagio finanziario, formando una nuova architettura di vigilanza che ha lo scopo di "proteggere il sistema finanziario europeo dai rischi futuri" (Consiglio dell'Unione Europea, 2009) e predisporre "nuovi strumenti comuni con i quali affrontare 
eventuali crisi finanziarie sistemiche" (Sole 24 Ore, 2016). Nel 2010 è stato istituito il SEVIF, Sistema europeo di vigilanza finanziaria, composto dall'European Systemic Risk Board, ESRB, l'European Banking Authority o EBA, l'European Insurance and Occupational Pensions Authority, EIOPA, l'European Securities and Markets Authority, ESMA.

Successivamente, a giugno 2012, i capi di Stato e di governo dei Paesi appartenenti all'Unione europea hanno varato il progetto di Unione bancaria cui partecipano gli Stati dell'UE che utilizzano la moneta unica o che hanno intrapreso l'iter per poterla adottare in futuro, nonché i Paesi che, pur non rientrando nell'Eurozona, hanno scelto di aderirvi mediante accordi di stretta cooperazione. L'Unione bancaria si prefigge di garantire la solidità degli intermediari finanziari e la loro capacità di superare eventuali crisi evitando il salvataggio delle banche in dissesto con denaro pubblico.

Da gennaio 2014, le previsioni degli organismi di vigilanza degli Stati appartenenti all'Unione Europea sono state integrate da quelle delle istituzioni di viglianza europee, alcune delle quali direttamente applicabili alle banche con sede nei singoli Paesi membri (BCBS, 2016). Il nuovo sistema normativo è stato codificato nel Single Rulebook o Codice unico europeo, costituito dalle previsioni di strumenti di prevenzione e gestione delle crisi bancarie, sistemi di garanzia dei depositi e requisiti patrimoniali per le banche.

In particolare, la direttiva 2013/36/UE, cosiddetta Capital Requirements Directive CRD IV e il regolamento UE n. 575/2013 Capital Requirements Regulation CRR recepiscono gli accordi di Basilea III sugli obblighi di capitalizzazione degli istituti finanziari. A tali nuove fonti normative si affiancano la direttiva sul sistema di garanzia dei depositi e la direttiva sul risanamento e la risoluzione delle banche o BRRD. Il quadro normativo si completa con le misure di esecuzione contenute nelle norme tecniche di regolamentazione o di attuazione, Regulatory Technical Standards RTS e Implementing Technical Standards ITS, adottate dalla Commissione europea su proposta dell'European Banking Authority EBA.

Mentre la CRD IV, la cui attuazione è di competenza dei singoli Stati, è stata recepita nel nostro Paese nella Circolare della Banca d'Italia n. 285 del 17 dicembre 2013, il CRR, ai sensi dell'art. 288 del Trattato sul funzionamento dell'Unione europea, così come anche le norme tecniche, è obbligatorio in tutte le sue parti e direttamente applicabile in ciascuno degli Stati membri.

La funzione di vigilanza attualmente è svolta nel quadro del Meccanismo di vigilanza unico, Single Supervisory Mechanism SSM, istituito dal Regolamento 1024/2013 dell'Unione europea e pienamente operativo dal 4 novembre 2014 attraverso il Supervisory Board, cui partecipano i rappresentanti delle Autorità di vigilanza nazionali e 6 membri permanenti. Il Supervisory Board formula proposte per l'adozione di decisioni da parte del Consiglio direttivo della Banca Centrale Europea ed è l'organismo competente sulla verifica del rispetto delle regole e sugli interventi di vigilanza.

Il nuovo ordinamento europeo interessa circa 8.300 banche appartenenti all'area euro e prevede la vigilanza diretta della Banca Centrale Europea su 129 banche considerate significative (BCE, 2016). La BCE inoltre può avocare a sé l'esercizio della supervisione anche sulle banche che richiedono l'assistenza finanziaria dell'European Financial Stability Facility o dell'European Stability Mechanism. L'esercizio della vigilanza su tutte le altre banche è compito delle autorità nazionali, che devono comunque operare in base alle linee guida fornite dalla BCE. 


\section{Principali nodi del sistema bancario e regolamentazione finanziaria internazionale}

Il sistema finanziario globale è, per sua stessa natura, dinamico e aperto e risente dell'azione dei grandi nodi finanziari che ospitano le direzioni delle istituzioni finanziarie che agiscono a livello mondiale.

I collegamenti fra banche e intermediari finanziari che hanno sede all'interno dello spazio europeo si estendono spesso oltre i confini dell'Eurozona e dell'Unione bancaria formando una rete fra nodi con diversi livelli di importanza. Gli indicatori per la misurazione di tale rilevanza riflettono la dimensione delle banche, il loro grado di interconnessione, la mancanza di sostituti o infrastrutture finanziarie prontamente disponibili per i servizi che forniscono, l'operatività internazionale svolta (in più giurisdizioni) e la loro complessità. In base a questi parametri, nello spazio finanziario si possono individuare banche a rilevanza sistemica globale, così definite in relazione all'impatto che il loro fallimento potrebbe avere sul sistema finanziario internazionale e sull'economia in generale (BCBS, luglio 2013).

Tab. 1 - Presenza nei centri finanziari delle prime 30 banche a rilevanza sistemica per grandi partizioni e bucket.

\begin{tabular}{|c|c|c|c|c|c|c|c|c|c|c|c|c|c|c|c|c|c|}
\hline & & \multicolumn{4}{|c|}{ Stati Uniti } & \multicolumn{10}{|c|}{ Europa } & \multicolumn{2}{|c|}{ Asia } \\
\hline & & SF & $\mathrm{CL}$ & NY & BS & MD & ED & LN & PA & AM & BE & $\mathbf{Z U}$ & FR & ML & SK & BJ & TK \\
\hline \multirow{5}{*}{ bucket } & 5 & & & & & & & & & & & & & & & & \\
\hline & 4 & & & - & & & & $\bullet$ & & & & & & & & & \\
\hline & 3 & & & - & & & & $\bullet$ & $\bullet$ & & & & $\bullet$ & & & & \\
\hline & 2 & & $\bullet$ & $\bullet \bullet$ & & & & & & & & $\bullet$ & & & & & - \\
\hline & 1 & $\bullet$ & & $\bullet$ & $\bullet$ & $\bullet$ & $\bullet$ & $\bullet$ & $\bullet \bullet$ & $\bullet$ & $\bullet$ & & & $\bullet$ & $\bullet$ & $\bullet \bullet$ & $\bullet \bullet$ \\
\hline
\end{tabular}

\section{Legenda:}

\begin{tabular}{llll}
\hline AM Amsterdam & CL Charlotte & MD Madrid & SF San Francisco \\
BE Basilea & ED Edimburgo & ML Milano & SK Stoccolma \\
BJ Beijing - Pechino & FR Francoforte & NY New York & TK Tokyo \\
BS Boston & LN Londra & PA Parigi & ZU Zurigo
\end{tabular}

(Nostra elaborazione in base ai dati: Financial Stability Board, 2015. Fonte degli acronimi delle città: Taylor et Al., 2002, con lievi modifiche).

Le banche a rilevanza sistemica globale sono suddivise in cinque livelli d'importanza crescente da 1 a 5, detti bucket. Il bucket 5, attualmente risulta vuoto; nel bucket 4 si trovano JP Morgan Chase di New York e HSBC di Londra; nel bucket 3 ci sono: Citigroup (New York), Barclays (Londra), BNP Paribas (Parigi), Deutsche Bank (Francoforte); il bucket 2 comprende tre banche statunitensi: Bank of America (Charlotte), Goldman Sachs e Morgan Stanley (New York), una banca europea: Credit Suisse (Zurigo) e una asiatica: Mitsubishi UFJ FG (Tokyo); infine nel bucket 1 troviamo tre banche statunitensi: Wells Fargo (San Francisco), Bank of New York Mellon (New York), State Street (Boston), in Europa: Santander (Madrid), Royal Bank of Scotland 
(Edimburgo), Standard Chartered (Londra), tre banche francesi con sede a Parigi: BPCE, Crédit Agricole e Société Générale, ING Bank (Amsterdam), UBS (Basilea), Unicredit Group (Milano), Nordea (Stoccolma), quattro banche cinesi: Agricultural Bank of China, Bank of China, China Construction Bank, Industrial and Commercial Bank of China Limited, tutte con sede a Pechino (Beijing) e due banche giapponesi con sede a Tokyo: Mizhuo e Sumitomo Mitsui.

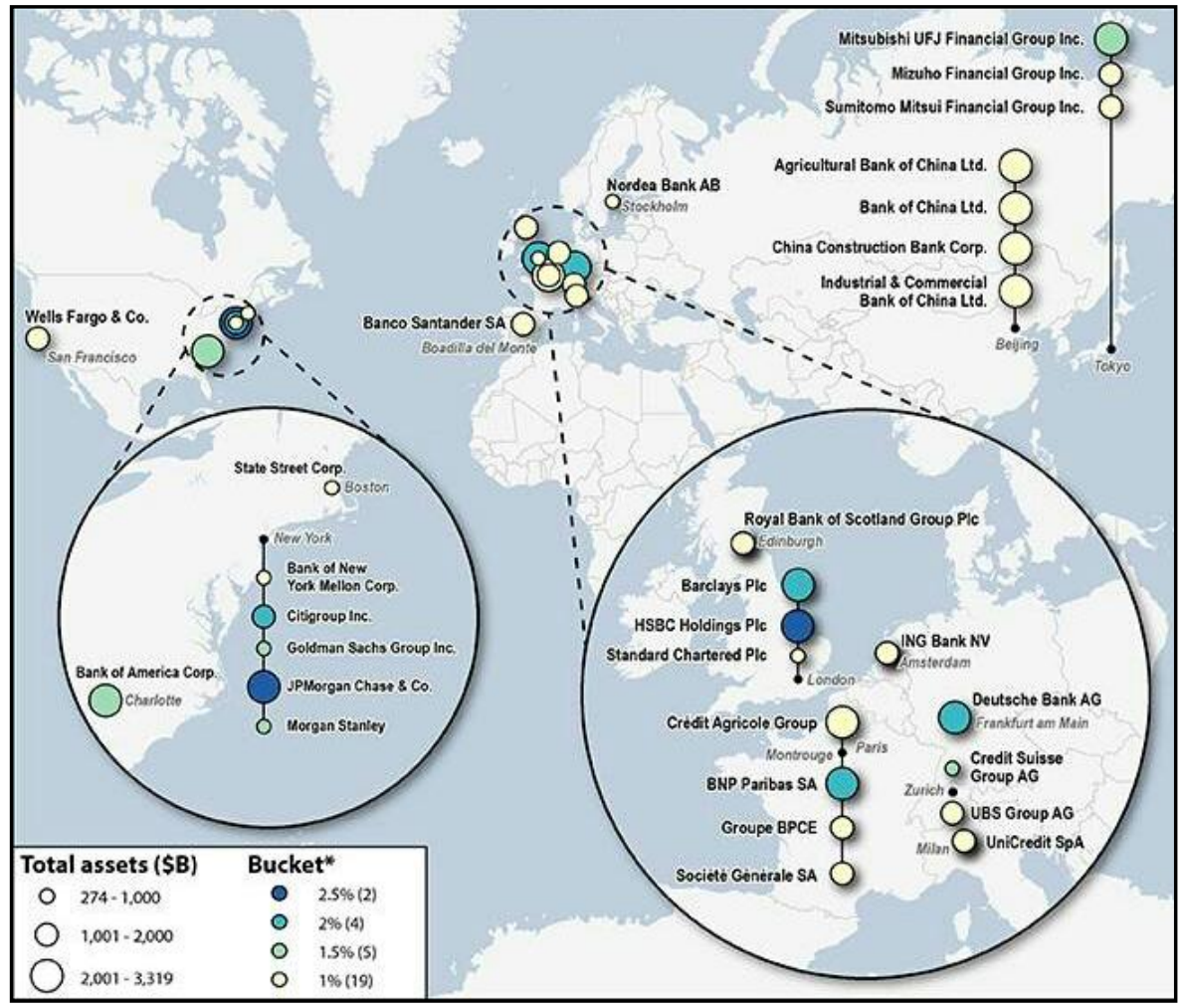

Fig. 5 - Polarizzazione delle banche a rilevanza sistemica per continente (fonte: Fox et Al., http:/ / mmw. financeasia.com - fonte dei dati: Financial Stability Board, 2015).

La tabella 1 riporta la distribuzione delle banche a rilevanza sistemica per centri finanziari e bucket, indicandole con punti di diversa entità in base ai parametri considerati. Si può osservare la loro presenza all'interno del confine dell'Eurozona e la concentrazione in tre poli principali in Europa, Stati Uniti d'America e Asia (cfr. fig. 5).

Gli eventuali aspetti di vulnerabilità dei nodi dello spazio finanziario globale costituiscono altrettanti fattori di rischio per la stabilità del sistema e dell'economia mondiale, pertanto la regolamentazione finanziaria emanata dalle istituzioni internazionali ha come principali destinatari proprio tali nodi.

In particolare, il Comitato di Basilea per la Supervisione delle Banche, Basel Committee on Banking Supervision BCBS, ha elaborato gli standard quantitativi e qualitativi che le banche a rilevanza sistemica sono tenute a rispettare come, ad esempio, previsioni 
di capitale che garantiscano la copertura delle potenziali perdite in bilancio conseguenti al deterioramento dei crediti o ad altri potenziali fattori di shock.

La graduazione dei parametri cui le banche devono uniformarsi sono stabiliti e revisionati periodicamente sulla base di un comprehensive assessment, ovvero di un processo di valutazione suddiviso in tre fasi: un supervisory risk assessment, ossia un'analisi dei profili di rischio, un asset quality review $\mathrm{O} \mathrm{AQR}$, con cui si esamina la qualità degli attivi di bilancio e uno stress test, che consiste nella verifica della capacità di ciascuna banca di continuare a operare in condizioni economiche sfavorevoli. Gli stress tests, come noto, si basano sulla simulazione delle variazioni dei valori dei rischi (sovrano, di credito, di controparte, di mercato, di liquidità) in ipotetici scenari economici avversi.

\section{Effetti della regolamentazione finanziaria sullo sviluppo sostenibile dei territori}

Quale sia la misura della dipendenza dello sviluppo economico dalle risorse fisiche e antropiche, materiali e immateriali, ereditate e create dai gruppi umani - presenti nei territori è una delle principali questioni cui i geografi hanno cercato di dare una risposta. Ad esempio, il clima, le condizioni naturali in cui si diffondono le epidemie oppure quelle più o meno favorevoli a determinate colture, le estrazioni di risorse minerarie, la valorizzazione di risorse paesaggistiche sono alcuni dei fattori fisicoantropici di sviluppo considerati.

Studi recenti hanno dimostrato, anche con metodi quantitativi, che le risorse naturali condizionano lo sviluppo economico in modo indiretto, ovvero che $\mathrm{i}$ fattori ambientali agiscono attraverso il loro impatto sulle istituzioni (Easterly e Levine, 2003) definite da alcuni autori come l'insieme delle regole formali (leggi, diritti, convenzioni) e informali (usi, costumi, tradizioni, codici di condotta, sanzioni, religioni, tabù) che consentono e limitano i comportamenti umani, modellando le relazioni economiche e sociali (North, 1991, Eggertsson, 1996). In questa prospettiva, nel processo di sviluppo regionale, si può esaminare la funzione di regolamentazione istituzionale considerando il legame fra istituzioni e spazio finanziario (Fernández e Tamayo, 2015).

In particolare, in riferimento agli effetti della regolamentazione finanziaria sullo sviluppo sostenibile, questi possono essere osservati considerando due processi chiave che hanno luogo nello spazio finanziario: il trasferimento dei rischi e la diffusione di liquidità (Diamond e Dybvig, 1983).

Oggi si assiste a una deformazione del tradizionale ruolo delle banche di assorbire il rischio dei risparmiatori, assumendolo insieme ai soggetti finanziati, in favore di un processo inverso di diffusione dei rischi dei soggetti finanziati sui detentori di strumenti di risparmio.

Un'evidenza di tale cambiamento si è avuta durante la crisi finanziaria del 2007, con la securitization o cartolarizzazione dei mutui, che "consiste nel trasformare un'attività finanziaria ... un credito derivante da un contratto di finanziamento ipotecario, o mutuo per l'acquisto della casa, in un'attività divisa e vendibile, ovvero in titoli. La società finanziaria che ha erogato un certo numero di mutui può decidere di 'cartolarizzare' tali crediti, emettendo e vendendo ad investitori privati o istituzionali dei titoli” che avranno un valore di mercato legato ai rimborsi delle rate dei mutui sottostanti, quindi ai pagamenti degli originari mutuatari. "Con il processo di securitization o 'cartolarizzazione', 
il rischio del mancato pagamento delle rate dei mutui è in certa misura incorporato nei titoli" e trasferito sui territori attraverso gli investitori istituzionali e i risparmiatori (Cesari, 2014).

Quanto alla relazione fra liquidità e sviluppo è necessaria una breve premessa. Nonostante non si possa prescindere dalla considerazione che regioni con lo stesso livello di prodotto interno lordo pro capite possano avere differenti caratteristiche in relazione a parametri come qualità e aspettativa di vita, scolarizzazione, accesso all'educazione e opportunità d'impiego, il prodotto interno lordo pro capite può essere considerato la componente di maggior peso nell'analisi quantitativa dello sviluppo sostenibile dei territori (Graeme, 2016).

La correlazione positiva fra prodotto interno lordo e liquidità in circolazione, cui è stato dato ampio rilievo già nelle fonti classiche del pensiero economico (Smith, 1776), è confermata da studi recenti. In particolare, è stato misurato che a un aumento unitario di liquidità pro capite corrisponde un incremento di circa il 2,57\% del prodotto interno lordo pro capite, correlazione validata dall'assenza di nesso di causalità inversa, ovvero dalla mancanza di relazione causa-effetto fra aumento del prodotto interno lordo e incremento della liquidità.

Tale correlazione positiva si realizza con un meccanismo di trasmissione che agisce prevalentemente attraverso banche e imprese. La concessione di prestiti da parte delle banche, specie alle piccole imprese, o a clienti senza opportunità di finanziarsi direttamente sul mercato dei capitali, assicura agli imprenditori la disponibilità di risorse finanziarie e consente la prosecuzione degli investimenti e l'espansione dell'attività economica (Berger, Sedunov, 2016).

In questa prospettiva, assumono importanza anche le trasformazioni del sistema di barriere istituzionali al contagio finanziario. La vigilanza bancaria, in particolare, si è tradizionalmente basata sulla previsione di percentuali di capitale che le banche devono detenere in misura proporzionale all'attività svolta, al fine di garantirne la stabilità. Poiché non si riscontra alcuna dipendenza significativa fra aumento del capitale - o del totale dell'attivo - delle banche e aumento della produzione, non si evidenziano effetti diretti di tali norme sullo sviluppo. Inoltre, nel momento in cui le banche emettono azioni o quote di capitale e si finanziano sul mercato con obbligazioni, la liquidità viene, almeno in una prima fase, drenata dal sistema economico in cambio titoli illiquidi.

Per compensare in parte questo meccanismo ed evitare il fenomeno della contrazione dei crediti, il cosiddetto effetto di credit crunch, le istituzioni finanziarie internazionali hanno definito nuovi interventi a fronte del rischio di liquidità. Con gli accordi di Basilea III, fra l'altro, sono stati introdotti due coefficienti minimi di liquidità: il Net Stable Funding Ratio e il Liquidity Coverage Ratio. Il primo è un indicatore di mediolungo periodo, che entrerà in vigore nel 2018, per il quale le banche dovranno aumentare la propria provvista stabile di liquidità. Il secondo è un indice di breve periodo, entrato in vigore nel 2015, e misura lo stock di risorse liquide necessario a ciascuna banca per fronteggiare, per un periodo della durata di almeno un mese, accentuati deflussi di liquidità dovuti, ad esempio, ad una "corsa agli sportelli" dei clienti, senza dover ricorrere a rifinanziamento né presso la Banca Centrale, né presso il mercato interbancario. In un possibile scenario di crisi di liquidità, si prevede che, attraverso il rispetto di questo parametro, le banche debbano avere riserve liquide sufficienti a restare autonome per 
almeno 30 giorni, ovvero per il tempo minimo necessario alle autorità monetarie per intervenire con manovre di più ampio respiro (Toniolo, 2015).

Alcuni autori, pur riconoscendo la correlazione fra liquidità e sviluppo, ritengono che queste misure di regolamentazione finanziaria non siano strettamente correlate alla capacità delle banche di creare liquidità e, di conseguenza, non abbiano impatti significativi sulle regioni (Berger, Sedunov, 2016). Si ritiene, inoltre, che un aumento degli indici di liquidità perseguito senza la previsione di un incremento della quota di prestiti a breve termine concessa alle imprese non possa avere effetti positivi sul territorio. Si valuta, al contempo, come non si possa prescindere nel lungo periodo dal ruolo delle istituzioni pubbliche per una definizione puntuale e costante di programmi per lo sviluppo di infrastrutture a sostegno dei settori finanziati dalle banche.

\section{Principali risultati e considerazioni finali}

Nello spazio finanziario, come detto, le aree interessate non seguono logiche di contiguità e continuità territoriale, ma gli ambiti regionali "si configurano come una rete di spazi variamente interconnessi, sottoposti nel tempo a trasformazioni che possono interessare estensione e attivazione delle connessioni e delle interdipendenze fra i suoi nodi", estendendosi oltre il confine delle aree sottoposte a regolamentazione e vigilanza da parte delle istituzioni (Cesari, 2014).

La presenza di confini politici e il sistema di barriere di regolamentazione e vigilanza istituzionale non costituiscono uno schermo al rischio di contagio finanziario proveniente dall'esterno e dall'interno dei confini, attraverso le connessioni delle banche a rilevanza sistemica presenti nell'Unione bancaria con il resto del sistema finanziario mondiale. A partire da tali nodi rilevanti, i rischi del sistema finanziario possono diffondersi e disperdersi, raggiungendo le intersezioni con i territori dell'economia reale e compromettendo gli equilibri economici e sociali e lo sviluppo sostenibile delle regioni.

In considerazione della necessità di favorire relazioni sostenibili fra finanza ed economia reale, gli effetti della regolamentazione finanziaria assumono grande rilevanza. La ricerca di soluzioni che non si limitino a gestire l'emergenza di fronte ad una nuova possibile crisi finanziaria non può prescindere dallo studio degli effetti della regolamentazione finanziaria sullo sviluppo.

L'attuale architettura di vigilanza è pensata per assicurare la stabilità dei nodi dello spazio finanziario tuttavia, ad oggi, non costituisce una barriera efficace alla diffusione dei rischi che si ripercuotono sui territori. Si ritiene perciò importante il rafforzamento del ruolo delle autorità di controllo sulla trasparenza sia dei prodotti finanziari sia del loro collocamento presso i risparmiatori. Sono infatti questi ultimi che, colpiti dalle crisi finanziarie, perdono la loro capacità di investimento producendo un indebolimento del tessuto economico che, se prolungato nel tempo, debilita le aree colpite impedendo prima la crescita e innescando poi successive regressioni nei livelli di sviluppo regionale prima alla scala locale e poi agli ambiti spaziali via via più grandi.

Si ritiene, inoltre, che un aumento degli indici di liquidità perseguito senza la contestuale previsione di incremento della quota di prestiti alle imprese, pur determinando una maggiore temporanea autonomia delle banche nella provvista di fondi, non abbia effetti positivi diretti sulla liquidità presente localmente, pertanto restano 
necessari previsioni e programmi di sviluppo, in particolare sostenibile, delle infrastrutture a sostegno dei settori finanziati dalle banche.

\section{Nota Bibliografica}

Aalbers M. (2009), "Geographies of the financial crisis". Area, 41 (1), pp. 34-42.

Agnes P. (2000), "The "end of Geography" in financial services? Local embeddedness and territorialization in the interest rate swaps industry". Economic Geography, 76 (4), pp. 347-366.

Albrechts L., Coppens T. (2003), "Megacorridors: striking a balance between the space of flows and the space of places". Journal of Transport Geography, 11, pp. 215-224.

Banca d'Italia. Vigilanza sul sistema bancario e finanziario (2016, aprile). www.bancaditalia.it/compiti/vigilanza/index.html

BCBS - Basel Committee on Banking Supervision. Bank for International Settlements (2016). Regulatory Consistency Assessment Programme (RCAP). Assessment of Basel III G-SIB framework and review of D-SIB frameworks - European Union. www.bis.org/bcbs/publ/d372.pdf

BCBS - Basel Committee on Banking Supervision. Bank for International Settlements (2013, luglio). Global systemically important banks: updated assessment methodology and the higher loss absorbency requirement. www.bis.org/publ/bcbs255.pdf

BCBS - Basel Committee on Banking Supervision. Bank for International Settlements (2013, gennaio), Il Liquidity Coverage Ratio e gli strumenti di monitoraggio del rischio di liquidità. www.bis.org/publ/bcbs238_it

BCE (2016). List of supervised entities. www.bankingsupervision.europa.eu/ecb/pub/pdf/list_of_supervised_entities

BCE (2016, aprile). Rapporto annuale 2015. www.ecb.europa.eu/pdf/ar2015it

Beaverstock J.V., Taylor P.J., Smith R.G (1999), “A roster of world cities”. Cities, 16 (6), pp. 445-458.

Berger A.N., Sedunov J. (2016), "Bank liquidity creation and real economic output". Social Science Research Network. papers.ssrn.com/sol3/papers.cfm?abstract_id=2515361

Bissanti A.A. (1974), "Applicazioni metacartografiche nella ricerca e nella didattica della geografia antropica". Bollettino dell' Associazione Italiana di Cartografia, 32, pp. 113-127.

Caselli G.P. (2014), "La Russia nella prima e seconda globalizzazione. Differenze e similarità". European Journal of Sustainable Development, 3 (2), pp. 41-52.

Castells M. (1996), "The information age: economy, society and culture". Vol. 1: The rise of the network society. Oxford, Blackwell.

Cesari O. (2014), "Periferie e centri finanziari. Origine locale della crisi mondiale?". In Fiori M., Varraso I. (Eds.), Periferie d'oggi e nuove realtà territoriali. Riflessioni ed esempi. Bari, WIP Edizioni, pp. 41-57.

Clark G.L. (2015), “The Geography of the European Central Bank: form, functions and legitimacy". Journal of Economic Geography, 15, pp. 855-881.

Consiglio dell'Unione Europea (2009), Consiglio Europeo di Bruxelles 18 e 19 giugno 2009. Conclusioni della Presidenza. Nota di trasmissione della Presidenza alle Delegazioni. www.consilium.europa.eu

Consiglio dell'Unione europea (2016, marzo), Unione bancaria. www.consilium.europa.eu

Diamond D.W., Dybvig P.H. (1983), "Bank runs, deposit insurance, and liquidity". The Journal of Political Economy, 91(3), pp. 401-419.

Dixon A.D. (2011), "The Geography of finance: form and function". Geography Compass, 11 (5), pp. 851-862.

Easterly W., Levine R. (2003), "Tropics, germs and crops: how endowments influence economic development". Journal of Monetary Economics, 50, pp. 3-39.

Eggertsson T. (1996), "A note on the economics of institutions". In Alston L.J., Eggertsson T., North D.C. (Eds.), Empirical studies in institutional change. Cambridge, Cambridge University Press, pp. 6-24.

Fernández A., Tamayo C.E. (2015), "From institutions to financial development and growth: what are the links?". Journal of Economic Surveys, 00 (0), pp. 1-41.

Financial Stability Board (2015, novembre), 2015 update of list of global systemically important banks (G-SIBs). www.fsb.org

Fiori M. (1989), "Percezione ambientale e carte mentali", in Valussi G. (Ed.), Friuli Venezia Giulia: regione problema. Aggiornamenti scientifici e didattici, Roma, Assoc. Ital. Insegnanti di Geografia, pp. 190-205.

Fiori M. (2012), Identità territoriale per lo sviluppo e l'imprenditorialità. Bari, Wip. 
Fox Z., Vanderpool C., Tai S. (2015, novembre), "Global banks ready for TLAC requirements". SNL Financial. www.financeasia.com

Friedmann J. (1986), “The world city hypothesis". Development and Change, 17, pp. 69-83.

Giannelli A. (2004), Geografia del credito: spazio finanziario e comportamenti strategico-territoriali - Un'analisi a scala italiana e pugliese. Napoli, E.S.I.

Gehrig T. (2000), "Cities and the Geography of financial centers" in Huriot J.M., Thisse J.F. (Eds.). Economics of cities. Cambridge, Cambridge University Press.

Gould P.R. (1969), Spatial diffusion. In Association of American Geographers Resource Papers, 4, Washington D.C., Association of American Geographers, the Pennsylvania State University and the National Science Foundation.

Graeme B. (2016), "Development, the European Union and the financial crisis: assessing the picture". Journal of Financial Crime, 23 (2), pp. 441-464.

Graeme B. (2015), "Responsibility and accountability in the financial sector". In Rider B. (2015), Research handbook on international financial crime. Cheltenham Glos, UK e Northampton Massachusetts, USA, Edward Elgar Publishing, pp. 239-249.

Hägerstrand T. (1967), Innovation diffusion as a spatial process. Chicago, The University of Chicago Press.

Hall S. (2013), "Geographies of money and finance III. Financial circuits and the 'real economy" ". Progress in Human Geography, 37 (2), pp. 285-292.

Kindleberger C.P. (1974). The formation of financial centers: a study in comparative economic history, Princeton, Princeton University Press.

Lucia M.G. (Ed.) (2010), Lo spazio geografico dell'economia finanziaria, Torino, Celid.

Martin R. (1994), "Stateless monies, global financial integration and national autonomy: the end of Geography?", in Corbridge S., Thrift N., Martin R. (Eds.), Money, power and space. Cap. 6, Oxford, Blackwell, pp. 152-176.

Martin R. (2011), "The local geographies of the financial crisis: from the housing bubble to economic recession and beyond". Journal of Economic Geography. 11, pp. 587-618.

North D.C. (1991), "Institutions". The Journal of Economic Perspectives, 5 (1), pp. 97-112.

Paltalidis N., Gounopoulos D., Kizys R., Koutelidakis Y. (2015), "Trasmission channel of systemic risk and contagion in the European financial network". Journal of Banking and Finance, 61, pp. 536-552.

Sander H., Kleimeir S., Heuchemer S. ( 2016), "The resurgence of cultural borders during the financial crisis: the changing Geography of Eurozone cross-border depositing". Journal of Financial Stability, 24, pp. $12-26$.

Sarre P. (2007), "Understanding the Geography of international finance". Geography Compass, 1, pp. 10761096.

Seo B. (2011), “Geographies of finance: centers, flows, and relations”. Hitotsubashi Journal of Economics, 52, pp. 69-86.

Smith A. (1776) An inquiry into the nature and causes of the wealth of nations. In Cannan, E. (Ed.) (1976), London, Methuen \& Co. Ltd, www.econlib.org/library/Smith/smWN.html

Sole 24 Ore (2016, 27 giugno). "Unione Bancaria". Argomenti. Le parole chiave. argomenti.ilsole24ore.com

Sudana I P. (2015), "Sustainable development and reconceptualization of financial statements". Procedia Social and Behavioral Sciences, 211, pp. 157-162.

Taylor P.J. (2001), "Specification of the world city network". Geographical Analysis, 33 (2), pp. 181-194.

Taylor P.J., Walker D.R.F., Catalano G., Hoyler M. (2002), "Diversity and power in the world city network". Cities, 19 (4), pp. 231-241.

Taylor P.J. (2005), "New political geographies: global civil society and global governance through world city networks". Political Geography, 24, pp. 703-730.

Thrift N. (2001), sub voce "Finance, Geography of". International Encyclopedia of the Social \& Behavioral Sciences, pp. 5655-5657.

Toniolo G. (2015), Verso Basilea III. www.etrformazione.it.m.youtube.com/watch?v=8Z098IEWWoE

Varraso I. (2004), Turismo e dinamiche territoriali di sviluppo. Valorizzazione delle risorse e organizzazione sistemica degli spazi garganici. Napoli, E.S.I.

Varraso I. (2009), "Visualizzare il tempo nelle rappresentazioni cartografiche", in Copeta C. (Ed.), Cartografie, immagini, metafore. Ravenna, Longo Editore, pp. 107-113. 